\title{
Aplicación de la tomografía computarizada de haz cónico en el diagnóstico de síndrome de Eagle
}

\section{Application of the cone beam computed tomography in the diagnosis of Eagle syndrome}

\author{
Verbel Bohórquez J*, Gómez Arcila V**, Castellar Mendoza C***, \\ Díaz Caballero A****
}

\section{RESUMEN}

En la práctica odontológica, cada vez son más comunes las alteraciones con sintomatología dolorosa en la región maxilofacial que no son apropiadamente diagnosticadas, a causa del desconocimiento de síndromes craneofaciales y cervicofaríngeos, como el síndrome de Eagle. Este último se describe como el dolor orofacial relacionado con la elongación de la apófisis estiloide y/o calcificación del ligamento estilohioideo. La aparición de una patología asociada a tal estructura, está acompañada de otros síntomas como: disfonía, disfagia, dolor faríngeo, glositis, otalgia, tonsilitis, dolor facial, cefalea, odinofagia, dolor en la articulación temporomandibular e imposibilidad de realizar movimientos laterales del cuello. El presente artículo es una revisión bibliográfica cuyo objetivo consistió en describir los aspectos generales del Síndrome de Eagle y la aplicabilidad de ayudas imagenológicas actuales en su identificación y diagnóstico. Se realizó una búsqueda de literatura en bases de datos como: MEDLINE (Ovid), Pubmed, Science Direct, y scirus empleando descriptores como: Eagle syndrome, cone beam, styloid process, Eagle syndrome in dentistry. A partir de la búsqueda se seleccionaron 37 artículos. Finalmente se pudo concluir que entre los diversos métodos diagnósticos, el que permite un análisis detallado de los patrones radiográficos de síndrome de Eagle, es la tomografía computarizada de haz cónico (Cone Beam Coputed Tomography).

Palabras clave (DeCS Bireme): Patología, dolor orofacial, diagnóstico, radiografía.

\section{SUMMARY}

In dental practice, alterations with painful symptomatology located in the maxillofacial region, which are not being diagnosed correctly, are every time more common, due to lack of knowledge of craniofacial and cervicofaringeal syndromes, such as the Eagle Syndrome. The latter, makes part of the head and neck disorders, and is described as an orofacial pain associated with the elongation of the styloid process and/or stylohyoid ligament calcification. The appearance of a pathology associated with such a structure, is accompanied by other symptoms such as: dysphonia, dysphagia, sore throat, glossitis, otalgia, tonsillitis, facial pain, headache,

Grupo de Investigación GITOUC. Facultad de Odontología. Universidad de Cartagena. Campus de la Salud. Cartagena. Colombia.

* Odontóloga. Universidad de Cartagena. Joven investigador Colciencias 2013.

** Odontóloga. Universidad de Cartagena. Maestrante en Microbiología. Joven investigador Colciencias 2013.

*** Odontólogo. Universidad de Cartagena. Joven investigador Colciencias.

**** Odontólogo. Universidad de Cartagena. Especialista en Periodoncia Universidad Javeriana. Magíster en Educación, Universidad del Norte. Candidato a Doctor en Ciencias Biomédicas Universidad de Cartagena. Docente titular Universidad de Cartagena. Director grupo de investigaciones GITOUC. 
odynophagia, pain in the temporomandibular joint and inability to perform lateral movements of the neck. This article is a literature review aimed to describe the general aspects of Eagle syndrome and applicability of current imaging aids in their identification and diagnostics. Literature research was performed using databases such as MEDLINE (Ovid), PubMed, Science Direct, Scirus and using descriptors such as: Eagle syndrome, cone beam, styloid process, Eagle syndrome in dentistry. From that search, 36 articles were selected. Finally it was concluded that among the various diagnostic methods, the one which allows a detailed analysis of radiographic patterns of the Eagle syndrome is the cone beam computed tomography.

Key words (MeSH Database): Pathology, facial pain, diagnosis, x-rays..

Fecha de recepción: 25 de febrero de 2014.

Aceptado para publicación: 5 de marzo de 2014.

Verbel Bohórquez J, Gómez Arcila V, Castellar Mendoza C, Díaz Caballero A. Aplicación de la tomografía computarizada de haz cónico en el diagnóstico de síndrome de Eagle. Av. Odontoestomatol 2014; 30 (6): $315-$ 323.

\section{INTRODUCCIÓN}

El síndrome de Eagle (SE) es una alteración patológica de cabeza y cuello, que genera dolor orofacial producto de la elongación de la apófisis estiloides y/o calcificación del ligamento estilohioideo (1)_ENREF_1. Al conjunto formado por la apófisis estiloides y el ligamento estilohioideo frecuentemente se le denomina "complejo estilohioideo", los cuales tienen su origen embrionario en el cartílago del segundo arco faríngeo o arco hioideo (cartílago de Reichert) (2). El SE hace parte de los desordenes temporomandibulares, que a su vez se enmarcan en desordenes musculoesqueléticos, encerrando un conjunto de alteraciones craneofaciales de etiología multifactorial, referidos a la articulación temporomandibular; músculos masticatorios y cervicales así como a estructuras asociadas (3).

Entre los métodos diagnósticos de dicha alteración se encuentran los métodos clínicos y radiográficos. El diagnóstico clínico puede ser realizado a través del examen físico, por medio de la palpación del proceso estiloides en la fosa amigdalina, estimulando así, el desarrollo de una sintomatología dolorosa (4). El dolor se presenta difuso, localizado en la región parotídea o irradiado hacia las regiones de la nuca, pabellón auditivo y garganta (5). Sin embargo, el diagnóstico definitivo se define con la realización de un examen radiográfico, en el cual se debe observar la elongación y/o calcificación del complejo estilohi- oideo (6). Para esto las radiografías panorámicas, laterales y anteroposterior de cráneo son ampliamente usadas. Autores como Guimarães SMR et al (2006), sugieren el uso de las radiografías panorámicas, debido a que pueden visualizar bilateralmente los proceso estiloides con sus variaciones de tamaño y forma (7). No obstante, en la actualidad existen técnicas imagenológicas con mayor especificidad pues reproducen tridimensionalmente la zona de estudio, como es el caso de la tomografía axial computarizada (8).

El SE se considera una patología poco identificada en el diagnóstico odontológico, posiblemente debido a su baja prevalencia en la población o desconocimiento del profesional de la salud. El objetivo de la presente revisión consistió en describir los aspectos generales del síndrome de Eagle y la aplicabilidad de ayudas imagenológicas actuales en su identificación y diagnóstico.

\section{MÉTODOS}

Se realizó una búsqueda de la literatura en las bases de datos MEDLINE (Ovid), Pubmed, Science Direct, y scirus; empleando los siguientes descriptores del Medical Subject Headings (MeSH): Eagle syndrome, cone beam, styloid process, eagle syndrome in dentistry. En las búsquedas se empleó un descriptor a la vez o combinaciones de estos empleando el 
operador booleano "AND". Además, las búsquedas se filtraron con la finalidad de obtener artículos originales y reportes de casos publicados entre los años 2000 hasta 2013, en idioma inglés y/o español y free full text. A partir de esto se obtuvieron 37 artículos que cumplieron con los anteriores criterios. Además, se incluyeron tres artículos clásicos publicados entre los años 1937 a 1986 que son de gran importancia para la revisión.

\section{ANÁLISIS E INTEGRACIÓN DE LA INFORMACIÓN. DEFINICIÓN Y DATOS HISTÓRICOS}

El síndrome de Eagle, también conocido como estilalgia, síndrome estiloides, síndrome de la arteria carótida, síndrome del proceso estiloides elongado y osificado o neuralgia estilohioidea (9). Fue descrito por primera vez por Watt W. Eagle en 1937, quien lo define como la relación entre la elongación de la apófisis estiloides y/o la osificación del ligamento estilohioideo con cuadros sintomatológicos debido al dolor relacionado con los nervios craneales y sensoriales en la región de la orofaringe, el cuello y oído (10-12). Esta anomalía pasa históricamente por tres periodos distintos. El primer periodo tuvo inicio hace 360 años con el primer reporte de osificación del proceso estilohioideo en 1652 por Marchetti, seguido 200 años después por Lucke en 1870 . Weinlecher (1872) reportó por primera vez los síntomas clínicos pre y postoperatorios de la osificación de la apófisis estiloides. Sterling, en 1896, reportó un caso clínico de la apófisis estiloides elongada (1). En 1907, Dwight clasificó la anatomía del complejo estilohioideo con base en radiografías, encontrando osificaciones de este con sintomatología clínica. En 1932, Thigpen reportó once casos de procesos estilohioideos elongados. Finalmente, Eagle describió el síndrome en 1937 y reportó dos casos que se relacionaban con un proceso estiloides anormal con sintomatología faríngea y facial por irritación de las carótidas (13). El segundo periodo corresponde al desarrollo del diagnóstico radiográfico cuando Grossman correlacionó los dolores del complejo incluyendo disfagia, otalgia, estilalgia, cefalea, dolor en la articulación temporomandibular y varias formas de dolor facial con la elongación del proceso estiloides. El tercer periodo corresponde al advenimiento de la radiogra- fía panorámica y de la tomografía computarizada, las cuales permiten la visualización de diversas estructuras del complejo maxilofacial (14).

\section{ETIOPATOGENIA}

La patogenia está dada por la compresión del nervio glosofaríngeo y/o estructuras vasculares asociadas a causa de la apófisis alargada (15). En cuanto a la etiología, existen diferentes teorías que tratan de explicarlo. Steinmann (16) propuso tres teorías para justificar el proceso de osificación:

1. Teoría de la hiperplasia reactiva, la cual explica que una cirugía o una irritación crónica puede producir tendinitis, periostitis osificante u osteítis en el ligamento estilohioideo (17-19).

2. Teoría de la metaplasia reactiva: Asociada a una osificación del tejido fibrocartilaginoso del ligamento estilohioideo, casi siempre como respuesta a un traumatismo previo.

3. Teoría de la variación anatómica: Propone que la elongación temprana de la apófisis estiloides y la osificación del ligamento son variaciones anatómicas que ocurren sin la presencia de trauma previo.

Esta última podría explicar la aparición de la osificación en niños $(20,21)$.

Por otro lado, se explica que la causa de sintomatología dolorosa es debido a la relación de la cadena estilohioidea con estructuras anatómicas, tales como músculos, nervios, arterias, venas y fascia, y su aumento de longitud puede llevar a la irritación e inflamación de dichas estructuras (4,14,22-24). Existen algunos mecanismos que pueden explicar la causa de dolor del SE, como: secundario a irritación mecánica de la mucosa faríngea; extensión de las terminaciones nerviosas del V, VII, IX y X nervios craneales, producto de la fibrosis posterior a la amigdalectomía; fractura del ligamento estilohioideo calcificado, derivado de trauma cervical o movimiento brusco del cuello, con consecuente proliferación de tejido de granulación y que puede originar presión de las estructuras que están en torno del complejo estilohioideo; presión sobre la arteria carótida, con estimulación de la cadena simpática que implica la vaina carotídea; y alteraciones degenerativas 
en el tendón de inserción del músculo estilohioideo o tendinitis de inserción (25).

Para el diagnóstico diferencial se deben considerar los tres síndromes del dolor más común que pueden ser confundidos con el dolor odontogénico:

- Tendinitis temporal.

- Síndrome de Ernest (inflamación del ligamento estilomandibular).

- Neuralgia trigeminal atípica.

Esta última puede tener una localización intra y extraoral en el trayecto del nervio trigémino, con ataques paroxísticos de segundos a minutos, dolor de fondo más continuo, resultando menos probable la completa remisión del dolor (11). También se deben incluir neuralgias glosofaríngea y trigeminal, artritis temporal, síndrome de dolor miofascial, dolor secundario a terceros molares impactados, artritis cervical, tumores, síndrome de la arteria carótida interna y trastornos temporomandibulares (26).

\section{SÍNDROME DE EAGLE Y CLASIFICACIÓN}

Según la sintomatología, Eagle describe dos tipos de síndrome: el clásico o típico y el asociado a la arteria carotídea o atípico (27). El primero ocurre en pacientes con previa amigdalectomía y se relaciona con la extensión de las terminaciones nerviosas, principalmente del IX par craneal (glosofaríngeo), causando un dolor constante o sordo en la región faríngea que con frecuencia se irradia al oído con quejas de disfagia, sensación de cuerpo extraño en la garganta, aumento de salivación, trismus, y limitación de movimientos a la excursión lateral. El segundo estaría presente en pacientes amigdalectomizados o no. El dolor podría estar asociado a la presión y obstrucción de las fibras simpáticas perivasculares de la arteria carótida, por la elongación y osificación del complejo estilohioideo, ocasionando sintomatología como: dolor en regiones infraorbitaria, temporal, auricular, occipital y cuello (28).

La apófisis estiloides es una proyección ósea de 2 a $2,5 \mathrm{~cm}$, delgada, larga y cilíndrica del hueso temporal desde la superficie inferior de éste en la unión de las porciones petrosa y timpánica por debajo del meato auditivo externo y anterior al proceso mastoi- deo. Se encuentra situada normalmente entre las arterias carótida interna y externa y permite la inserción de los músculos estilofaríngeo, estilohioideo y estilogloso. Además, de los ligamentos estilohioideo y el estilomandibular (7); el primero se origina en la punta del proceso y se inserta en el cuerno o asta menor del hueso hioides; el segundo se origina en la cara medial del proceso y corre anteroinferiormente hacia la superficie interna del ángulo goníaco de la mandíbula (3). Eagle (12) afirma que la longitud normal de la apófisis estiloides es de aproximadamente $25 \mathrm{~mm}$, por lo tanto, cualquier longitud superior a ese tamaño sería considerado como elongado (Figura 1).

\section{Clasificación de la elongación y calcificación según Langlais (29)}

Robert P. Langlais et al (29), en 1986, realizaron una clasificación para los patrones de elongación y calci-

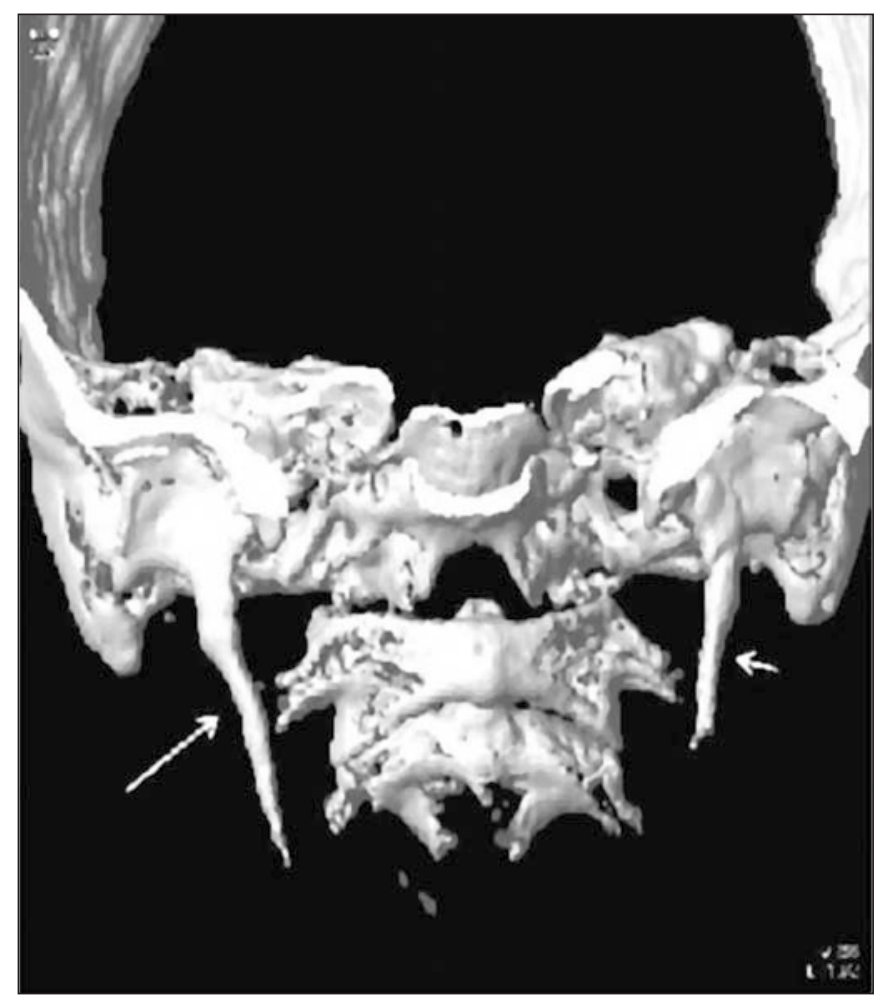

Fig. 1. Se observa apófisis estiloides elongada de forma bilateral, con medidas de 45,6 $\mathrm{mm}$ para el lado derecho y $37 \mathrm{~mm}$ en el lado izquierdo (tomado de Savranlar A, Uzun L, Birol M, Özer T. Threedimensional CT of Eagle's síndrome. Diagn Interv Radiol. 2005;11: 206-9). 
ficación del complejo estilohioideo, según su apariencia radiográfica. Actualmente se conocen 3 patrones radiográficos y 4 de calcificación.

- Tipo I o elongado: Este complejo estilohioideo calcificado, se caracteriza por una integridad ininterrumpida de la imagen estiloides. Si el estudio se realiza mediante la observación de radiografías panorámicas, se acepta como normal una longitud de $25 \mathrm{~mm}$ para la apófisis estiloides por la magnificación que sufren la mayoría de estas.

- Tipo II o pseudoarticulado: En este tipo de apariencia radiográfica, la apófisis estiloides está unida al ligamento estilomandibular o al ligamento estilohioideo por una sola pseudoarticulación, la cual se encuentra localizada superior al borde inferior de la mandíbula (de manera tangencial). Esto da la apariencia de un complejo articulado y elongado.

- Tipo III o segmentado: Consiste en porciones de la apófisis estiloides que son cortas o largas, observándose discontinuidad o segmentos interrumpidos del ligamento mineralizado. Se aprecian dos o más segmentos, con interrupciones ya sea por encima o debajo del borde inferior de la mandíbula, o ambos. La apariencia es de un complejo mineralizado y segmentado.

Teniendo en cuenta el patrón de calcificación se describe:

- Contorno calcificado: Se observa un borde radiopaco y delgado con una radiolucidez central que constituye la mayoría de la apófisis. Este patrón da la apariencia radiográfica de un hueso largo.

- Parcialmente calcificado: Indica que la apófisis tiene un contorno radiopaco y casi completamente opacificado, no obstante puede presentar centros discontinuos radiolúcidos.

- Complejo nodular: Se caracteriza por un contorno ondulado o festoneado. Puede estar parcial o completamente calcificado con diversos grados de radiolucidez central.

- Completamente calcificado: Es totalmente radiopaco, sin evidencia de radiolucidez interior.

\section{TRATAMIENTO}

El tratamiento del SE dependerá de la intensidad de los síntomas. En los casos en que la sintomatología es de media intensidad, no es recomendado ningún tratamiento, excepto tranquilizar al paciente; Algunos autores defienden el tratamiento clínico con analgésicos, antiinflamatorios, psicotrópicos, infiltración de corticoides o anestésicos. Sin embargo, en casos graves el tratamiento más efectivo y satisfactorio es la resección quirúrgica de la apófisis estiloides, ya sea por vía externa o intraoral. La vía intraoral, a pesar de ser más rápida y no dejar cicatriz externa visible, no permite una visualización adecuada de las estructuras adyacentes a la apófisis estiloides y representa un riesgo mayor de contaminación de los espacios cervicales. Las ventajas de la vía externa incluyen: mejor exposición y preservación de las estructuras vasculares y nerviosas, así como la resección más amplia de la apófisis estiloides. La cicatriz resultante de esta vía de abordaje es cervical alta, pequeña y estéticamente aceptables $(25,30)$.

\section{PREVALENCIA A NIVEL MUINDIAL}

Marissa R (2012) afirma que la prevalencia de un proceso estiloides alargado tiene gran variabilidad en la población. Eagle, en su publicación original, halló el proceso estiloides elongado en $4 \%$ de sus casos (20). De igual forma se señala que entre un $4 \%$ y $28 \%$ de la población posee procesos estiloides elongados, y de ellos, el $4 \%$ evidencia sintomatología dolorosa (3). Al realizar análisis de lateralidad, los hallazgos indican que es más frecuente encontrar elongaciones bilaterales (derecho e izquierdo), con mayor longitud en el lado derecho (4). Estudios realizados en países como Perú, Chile y México demuestran que es una patología que mayormente afecta a las mujeres en un rango de edad adulta de aproximadamente 60 a 70 años. La longitud promedio de elongación es de $30 \mathrm{~mm}$ a 37 $\mathrm{mm}$, siendo en los hombres los mayores valores encontrados en una población en Brasil $(27,31)$. En Santander, Colombia (3), en un estudio realizado por la asociación de otorrinolaringología en relación al síndrome de Eagle, se detectó que la variable sexo no fue estadísticamente significativa, y se concluyó que la técnica de evaluación de los procesos estiloides mediante tomografías computarizadas es el mejor recurso para medir adecuadamente esta proyección ósea, exponiendo a las personas a una dosis baja de radiación. 


\section{MÉTODO DIAGNÓSTICO RADIOGRÁFICO}

Diversas técnicas imagenológicas permiten demostrar la presencia de alargamiento y/o mineralización del proceso estilohioideo. No obstante, la tomografía axial computarizada de haz cónico (CBCT) se ha convertido en un método eficaz en la visualización de estructuras de cabeza y cuello, sin la presencia de superposiciones, y/o altos grados de distorsión. La CBCT incluye menor distorsión, mayor escala del contraste y eliminación del deslumbramiento (32-36) (Figura 2).

La palabra tomografía está formada por la unión de dos términos griegos "tomos" y "graphos" que significan respectivamente, partes y registro. De esta forma, la tomografía consiste en la obtención de imágenes del cuerpo en partes o cortes, y puede ser clasificada en dos tipos: tomografía convencional y tomografía computarizada (TC). Esta última se subdivide en tomografía computarizada médica y tomografía computarizada de haz cónico (CBCT).

La CBCT fue desarrollada a finales de los años noventa con el fin de obtener escáneres tridimensio-

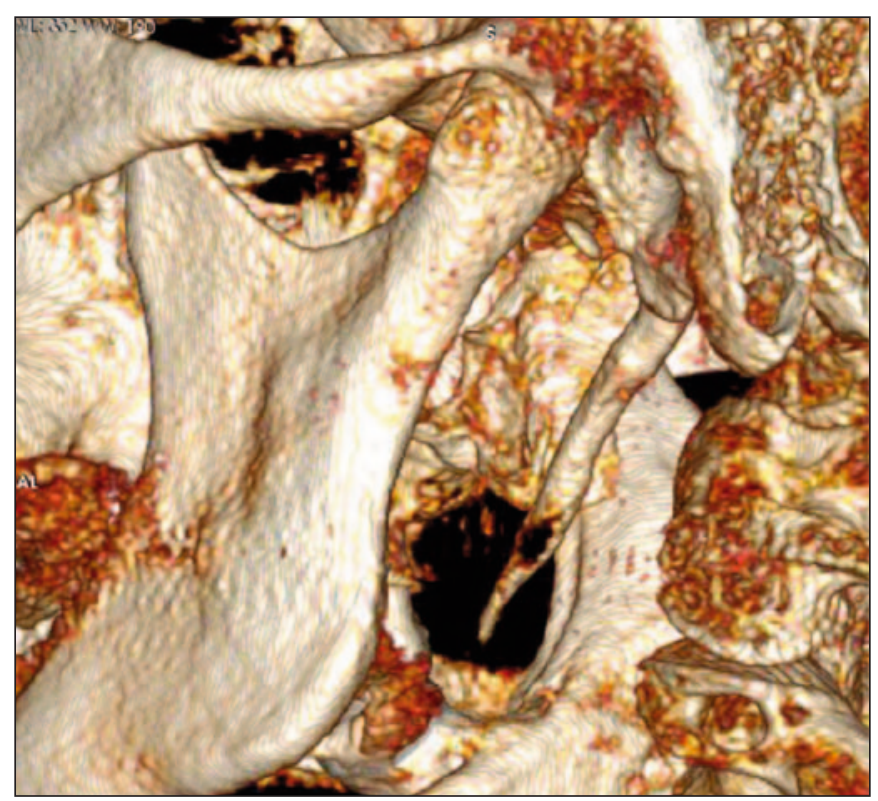

Fig. 2. Se evidencia CBCT-3D. Apófisis estiloides izquierda elongada (tomado de Mareque J, Hernández F, Biosca M, Coll M, Arenaz J. Abordaje intraoral en el síndrome de Eagle. Presentación de un caso clínico. Revista Española de Cirugía Oral y Maxilofacial. 2011:41-5). nales del esqueleto maxilofacial con una dosis de radiación menor que la tomografía computarizada médica o convencional, revolucionando la imagen del complejo craneofacial y ofreciendo una alternativa a la imagen convencional intraoral y panorámica, que elude superposición y los problemas de distorsión de imágenes (32,33) (Figuras 3 y 4). Sin embargo, al comparar las radiografías panorámicas convencionales con respecto a las reconstrucciones panorámicas

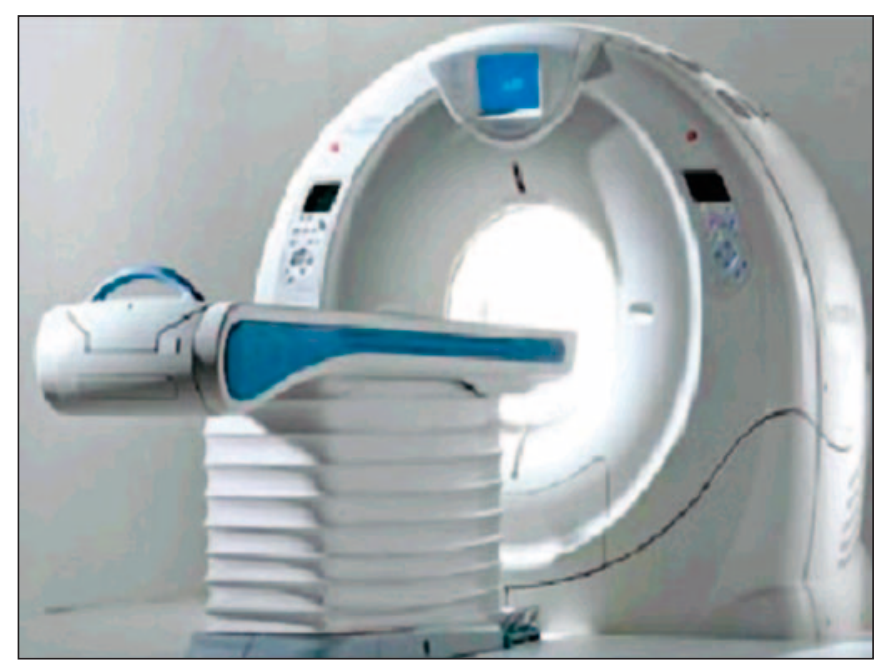

Fig. 3. Aparato TC médica de última generación (tomado de Lenguas A, Ortega R, Samara G, López M. Tomografía computarizada de haz cónico. Aplicaciones clínicas en odontología; comparación con otras técnicas. Cient Dent. 2010;7(2):147-59).

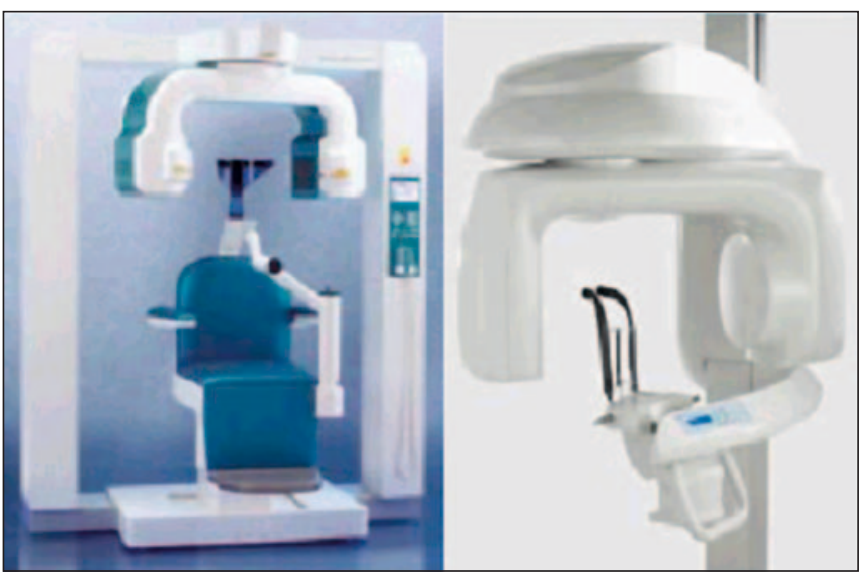

Fig. 4. Aparatos CBCT (tomado de Lenguas A, Ortega R, Samara G, López M. Tomografía computarizada de haz cónico. Aplicaciones clínicas en odontología; comparación con otras técnicas. Cient Dent. 2010;7(2):147-59). 
obtenidas a partir de la CBCT se observa que, con la imagen que se obtiene en la CBCT, se evitan los artefactos que aparecen siempre con la radiografía convencional, como puede ser la superposición de la columna vertebral (32).

En la tomografía computarizada médica, las imágenes son capturadas en las pantallas del detector y están hechas de múltiples planos, hasta obtener una imagen completa, por lo que precisa mayor radiación al paciente. Se obtiene un escaneado secuencial y espiral. El escáner realiza múltiples giros y en cada giro toma una imagen. A diferencia de ésta, la tomografía computarizada de haz cónico emplea un haz de rayos en forma de cono que es más estrecho, el cual requiere una sola rotación del tubo de rayos $\mathrm{X}$ $194^{\circ}-360^{\circ}$, tomando en cada grado una o dos imágenes alrededor de la cabeza del paciente para adquirir directamente los datos en lugar de múltiples rotaciones como la TC médica $(32,37)$ (Figura 5).

\section{CONCLUSIÓN}

La tomografía computarizada de haz cónico es una herramienta útil y eficaz en el diagnóstico de condiciones patológicas óseas y dentales. Muchos son los pacientes que previa consulta odontológica, ya pasaron por otras especialidades médicas sin haber conseguido el diagnóstico correcto. Es por esto, que

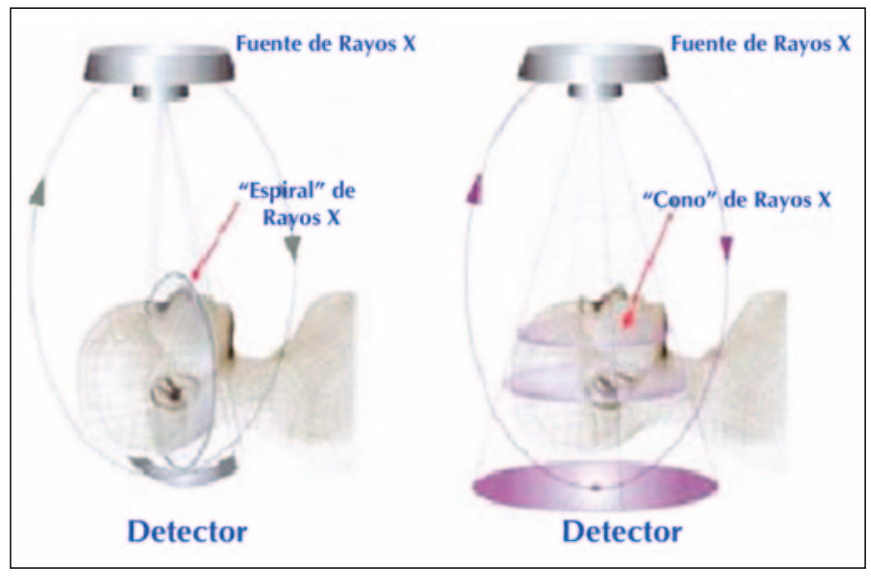

Fig. 5. Escaneado tipo haz plano o espiral y escaneado tipo haz cónico (tomado de Martínez N, Paredes V, Ortiz R, Gandía JL.

Funcionamiento de la TC médica y de la TC de haz cónico en odontología. ¿Qué debemos saber? Rev Esp Ortod. 2011;41:31-7). la detección temprana del síndrome de Eagle por medio de soportes físicos y radiográficos es importante para detener el desarrollo de la sintomatología que en su mayoría es dolorosa y vaga, y así facilitar un tratamiento oportuno evitando futuras complicaciones neurológicas, físicas, emocionales y psicológicas por la severidad del dolor.

Por tanto, la CBCT, al ser un método fiable y actual, permitirá el análisis detallado de los patrones de elongación y calcificación del complejo estilohioideo. Sin embargo, pese a su limitante de asequibilidad debido a su alto costo, existe la opción a través del programa o software de CBCT, digitalizar radiografías convencionales, obteniendo imágenes bidimensionales, lo cual es un avance tecnológico en el campo de la odontología.

\section{AGRADECIMIENTOS}

Johana Verbel, Verónica Gómez, Camilo Castellar agradecen al Departamento Administrativo de Ciencia, Tecnología e Innovación - Colciencias- por su programa "Jóvenes investigadores".

\section{BIBLIOGRAFÍA}

1. Ladeira F, Iwaki L. Styloid - Stylohiod sindrome: Literature review and case report. J Oral Maxillofacial Surg 2007;65:1346-56.

2. Sadler TW, Langman J. Embriología médica: con orientación clínica. $10^{\mathrm{a}}$ ed. impreso en Chile: Ed. Médica Panamericana 2007. 387 p.

3. Castillo C, Mantilla J, Sandoval G, Ramírez L. Síndrome de Eagle: Tomografía del proceso estiloídeo elongado. Acta De Otorrinolaringología y Cirugía de Cabeza y Cuello 2003;31(3):89-96.

4. Da Silva HJ, Arruda de Moraes SR, Tashiro T. Análisis morfométrico de los elementos de la cadena estilohioídea. Revista chilena de anatomía 2002;20(2):205-10.

5. Fini G, Gasparini G, Filippini F, Becelli R, Marcotullio D. The long styloid process syndrome 
or Eagle's syndrome. Journal of cranio-maxillofacial surgery 2000;28(2):123-7.

6. Orhan K, Güldiken Y, Ural H, Cakmak A. Elongated styloid process (Eagle's syndrome): literature review and a case report. Ağr1 2005;17 (2):23-5.

7. Guimarães SMR, Peixoto BC, Gomes MB, Carvalho ACP, Guimarães JP. Prevalência de alteração morfológica do processo estilóide em pacientes com desordem temporomandibular. Radiol Bras 2006;39(6):407-11.

8. Scarfe WC, Farman AG, Sukovic P. Clinical applications of cone-beam computed tomography in dental practice. Journal-Canadian Dental Association 2006;72(1):75.

9. Reis S, Carvalho P, Reis H. Processo estilóide alongado - relato de dois casos. Jornal Brasileiro de Oclusão, ATM e Dor Orofacial 2001;1(4):296300.

10. Lima Junior J, Rocha J, Ribeiro E, Costa V, De Sousa E. Síndrome de Eagle: revisión de la literatura. Acta Odontol Venez 2007;45(2):290-3.

11. Hernández C, Rodríguez M, Sano R, Vargas S, Monasterio M. Síndrome de Eagle: a propósito de un caso. Acta Otorrinolaringol 2000;12(2):57-60.

12. Eagle W. Elongated styloid process. Report of two cases. Arch Otolaryngol. 1937;25:548-587.

13. More CB, Asrani MK. Evaluation of the styloid process on digital panoramic radiographs. The Indian journal of radiology $\varepsilon$ imaging 2010;20(4): 261-5.

14. Domingues A, Zardo M, Oliveira A, Pires R, Barros F, De Oliveira M, et al. Alongamento do processo estilóide (síndrome de Eagle): relato de dois casos. Radiol Bras 2004;37(5):385-7.

15. Varelas PN, Sinson G, Rand S, Book D. Clipping the Eagle's wings: Treatment of an unusual cause of transient cerebral ischemia. Neurology 2005;64 (2):393-4.
16. Steinmann EP. A new light on the pathogenesis of the styloid syndrome. Archives of Otolaryngology -Head E Neck Surgery 1970;91(2):171.

17. Mendelsohn AH, Berke GS, Chhetri DK. Heterogeneity in the clinical presentation of eagle's syndrome. Otolaryngology-Head and Neck Surgery 2006;134(3):389-93.

18. Mortellaro C, Biancucci P, Picciolo G, Vercellino V. Eagle's syndrome: importance of a corrected diagnosis and adequate surgical treatment. Journal of Craniofacial Surgery 2002;13(6):755-8.

19. Rezgui-Marhoul L, Douira W, Saïd W, Bouslama K, Ben Dridi M, Hendaoui L. Le syndrome de Eagle: à propos d'un cas. Revue de Stomatologie et de Chirurgie Maxillo-faciale 2004;105(1):50-2.

20. Marissa R. Síndrome Eagle: reporte de un caso. Odontoestomatología. 2012;14(20):26-31.

21. López MC, Galdames IS, Rojas BV, Matamala DZ, Cortés RM, CANTíN L, et al. Síndrome de Eagle bilateral, reporte de un caso. Int J Odontostomat 2007;1(2):141-5.

22. Ramírez L, Sandoval G, Ballesteros L. TDM and head symptomatology. Med Oral Patol Cir Bucal. 2005;10:E18-E25.

23. Anselmo-Lima W, Rocha E, Souza H, Rodrigues C, Aparecido de Oliveira L. Alongamiento do proceso estiloide. A propósito de quatro casos. Rev Bras, de Otorrinolaringología 2005;58(2):124-9.

24. Harster P. DTM y estiloides. Let me do some speculation about stylohidal process. Ideas y Trabajos Odontoestomatológicos 2001;2(2):85-9.

25. Tiago RSL, Marques Filho M, Maia CAS, Santos OFS. Síndrome de Eagle: avaliação do tratamento cirúrgico. Rev Bras Otorrinolaringol 2002;68(2): 196-201.

26. Quereshy FA, Gold ES, Arnold J, Powers MP. Eagle's syndrome in an 11-year-old patient. Journal of oral and maxillofacial surgery 2001;59 (1):94-7. 
27. Politi M, Toro C, Tenani G. A Rare Cause for Cervical Pain: Eagle's Syndrome. International journal of dentistry 2008;2009:1-3.

28. Kosar M, Atalar M, Sabanclogullari V, Tetiker H, Erdil F, Cimen M, et al. Evaluation of the length and angulation of the styloid process in the patient with pre-diagnosis of Eagle syndrome. Folia morphologica. 2011;70(4):295-9.

29. Langlais RP, Miles DA, Van Dis ML. Elongated and mineralized stylohyoid ligament complex: a proposed classification and report of a case of Eagle's syndrome. Oral surgery, oral medicine, oral pathology 1986;61(5):527-32.

30. De Farias J, Flores P, Ribeiro V, Jose M, Correia M. Síndrome de Eagle. Revista Brasileira de Ciências da Saúde 2006;10(1):99-102.

31. Buono U, Mangone GM, Michelotti A, Longo F, Califano L. Surgical approach to the Stylohyoid Process in Eagle's Syndrome. Journal of oral and maxillofacial surgery 2005;63(5):714-6.

32. Martínez NZ, Gallardo VP, Ortiz RMC, Franco AYJLG. Funcionamiento de la TC médica y de la TC de haz cónico en odontología. ¿Qué debemos saber? Rev Esp Ortod. 2011;41:31-37.

33. Ronda N. Aplicaciones de la TAC en endodoncia. Electronic Journal of Endodontics Rosario 2012;2:635-62.
34. Adibi S. Cone Beam Computed Tomography for General Dentists. Open Access Scientific Report 2012;1(11):1-5.

35. Bae M-J, Kim J-Y, Park J-T, Cha J-Y, Kim H-J, Yu $\mathrm{H}-\mathrm{S}$, et al. Accuracy of miniscrew surgical guides assessed from cone-beam computed tomography and digital models. American Journal of Orthodontics and Dentofacial Orthopedics 2013; 143(6):893-901.

36. Barghan S, Tetradis S, Nervina JM. Skeletal and soft-tissue incidental findings on cone-beam computed tomography images. American Journal of Orthodontics and Dentofacial Orthopedics 2013; 143(6):888-92.

37. Lenguas A, Ortega R, Samara G, López M. Tomografía computarizada de haz cónico. Aplicaciones clínicas en odontología; comparación con otras técnicas. Cient Dent 2010;7:147-59.

\section{CORRESPONDENCIA}

Antonio Díaz Caballero

Facultad de Odontología.

Universidad de Cartagena.

Campus de la Salud Zaragocilla.

Cartagena, Bolívar

Colombia

Correo electrónico: adiazc1@unicartagena.edu.co 